\title{
Towards Accurate Estimation of Fingerprint Ridge Orientation Using BPNN and Ternarization
}

\author{
Prasad Reddy P.V.G.D ${ }^{1}$, M. James Stephen ${ }^{2}$ \\ ${ }^{1}$ (Professor, Dept. of CS\&SE, Andhra University, Visakhapatnam - 530 003, India) \\ ${ }^{2}$ (Research Scholar, Department of CS \& SE, Andhra University, India)
}

\begin{abstract}
Accurate estimation of ridge orientation is a crucial step in fingerprint image enhancement because the performance of a minutiae extraction algorithm and matching heavily relies on the quality of the input fingerprint images, which in turn relies on proper estimation of ridge orientation. When ridge structure is not clear in a noisy fingerprint image, ridge orientation estimation becomes tough and it is considered as one of the most challenging tasks in fingerprint image enhancement process.

A new methodology based on neural network approach and Ternarization for ridge orientation estimation is proposed in this paper. In the present work a trained Back Propagation Neural Network (BPNN) is used for accurate ridge orientation. The advantage with the Ternarization process is that it eradicates the false ridge orientation for which the neural network wrongly responds with the larger value and at the same time, it keeps the correct ridge orientation blocks intact without making them blur. This helps in qualitative extraction of minutiae points from the fingerprint image. The experimental results have shown that the proposed method for estimating ridge orientation works far better than the traditional gradient based approach.
\end{abstract}

Keywords: Feature Vector, Minutiae Extraction, Neural Network, Ridge orientation, Ternarization,

\section{Introduction}

Ridge orientation estimation is a necessary and fundamental step in fingerprint image enhancement and recognition. Ridge orientation is the basic feature of a fingerprint image, and ridge orientation estimation can be considered almost as a prelude to fingerprint image enhancement and matching process.

Accurate estimation of ridge orientation is a crucial step in fingerprint image enhancement because the performance of a minutiae extraction algorithm and matching heavily relies on the quality of the input fingerprint images, which in turn relies on proper estimation of ridge orientation.

Generally in a gray-level fingerprint image, ridges and furrows in a local neighborhood form a sinusoidal-shaped plane wave which has a well defined frequency and orientation. Many techniques were proposed by taking advantage of this information to enhance gray scale fingerprint images [1], [2], [3], [4], [5].

However all these techniques work on the assumption that the 'local ridge orientations can be reliably estimated'. But in practice, this assumption is not valid for fingerprint images, which are of poor quality, which restricts the applicability of these techniques.

Since the ridge structure is not clear in a noisy fingerprint image, ridge orientation estimation becomes tough and it is considered as one of the most challenging tasks in fingerprint image enhancement process.

So in order to attend this problem, a new methodology based on neural network approach and Ternarization for ridge orientation estimation is proposed. In the present work a trained back propagation neural network is used for accurate ridge orientation. Though the proposed concept was published in [19], complete clarity was not given on Ternarization and feature vector. The major difference of the present paper with the earlier one is that here, only 12 orientations are taken in the present work and experimental results section demonstrates the superior performance of the proposed method over the other existing techniques. Many fingerprint image enhancement techniques were proposed and implemented [20] [21] [22] but it is observed that accurate estimation of fingerprint ridge orientation plays crucial role in fingerprint image enhancement and hence lot of effort is spent on studying and experimenting various ridge orientation estimation techniques.

In the proposed methodology, the image is first divided into blocks then for each image block, a feature vector $\left\langle\mathrm{F}_{1}, \mathrm{~F}_{2}, \ldots \ldots \mathrm{F}_{11}\right\rangle$ is computed to be fed into the network which will respond to the vector with a value as an output. The elements in the feature vector are derived through the important parameters and properties of the fingerprint image.

The trained neural network generally responds with large value to correct ridge orientation of ridge block (of high quality or manually recoverable) and responds with a small value to those blocks which contain no ridges or contain manually unrecoverable ridges or falsely estimated orientations. 
The responded value by the trained neural network to a specific block is depended on the orientation, because the items from $F_{5}$ to $F_{11}$ of the input vector $\left\langle F_{1}, F_{2}, \ldots . . F_{11}\right\rangle$ have a close relationship with the estimated ridge orientation.

In the present work, 12 different orientations are considered. So for each block, the input vector is calculated for each orientation and generates 12 input vectors (each for 12 orientations). Those 12 vectors are fed to the network and obtain 12 responded values, respectively. Whichever orientation vector gets the highest value, that orientation is considered as the true orientation.

The similar methodology of estimating ridge orientation is presented in [6], where 16 different orientations are taken. The major drawback in that method is the usage of 2D low-pass filter in the image domain. Sometimes it is possible that the neural network may return large responded value for the low quality ridge blocks on some false orientations. To reduce this effect, in [6] a 2-dimensional low-pass filter is used, which makes the image more blurred. It affects correct orientation blocks also because the low-pass filter is applied on the image domain.

In the proposed methodology, a new concept, known as Ternarization is used to reduce this effect of false orientations of low quality ridge blocks with high responded value. Ternarization is the process dividing the image block into three blocks. They are called white blocks, black blocks and gray blocks. White blocks consist the ridge blocks with true ridge orientation with high responded values (point of interest). Black block consists ridge blocks with false orientations with low responded values (out of interest). Gray block consists the blocks with uncertainty (probably the false orientation ridge blocks with high responded value). Without affecting the true orientation fields the false orientation fields will be removed through the process of Ternarization. During the process the gray block becomes empty by throwing its elements either to white block or black block.

The advantage with the Ternarization process is that it eradicates the false ridge orientation for which the neural network wrongly responds with the larger value and at the same time, it keeps the correct ridge orientation blocks intact without making them blur. This helps in qualitative extraction of minutiae points from the fingerprint image.

\section{Existing approaches for estimation of ridge orientation.}

There are already some methods for fingerprint ridge orientation estimation in literatures. Most of them are based on relationship of pixel intensity between pixels [7]. Among them, the most popularly used method for ridge orientation estimation is the gradient-based method or it's variant. The gradient-based method is easy to be interfered by noise. In order to correct the false orientation caused by noise, [8] uses low pass filtering to smooth the orientation field. Sherlock [9] generates the orientation field based on the location of singularities, including core and delta. This method fails to distinguish two fingerprints which have the same locations and numbers of singularities but the different orientation field pattern. This method is improved by Vizcaya [10] and Araque [11]. However, low quality image block may contain edges which is not that of the ridges but of the noise caused by ridge interrupts, damp, dirt, etc. In order to correctly estimate ridge orientation in noisy ridge areas, a machine learning based method using neural network and Ternarization process is proposed.

\section{Proposed Methodology}

In an ideal fingerprint image, ridge and furrows alternate and flow in a locally constant direction. In such ideal cases ridges can be easily detected and minutiae can be precisely located. In this context the gradient based approaches works well for the purpose of ridge orientation estimation. But with poor quality fingerprint images and latent fingerprint images, these techniques fail to estimate ridge orientation properly. So a new methodology based on neural network approach and Ternarization is proposed for effective estimation ridge orientation.

The proposed methodology is based on the technique proposed by Zhu E., Yin J.P., et.al, [6]. In the present work 12 different orientations are used to give as input to the neural network. The training methodology is described in [12]. The major modification that is done to the technique mentioned in [6] is that in the present work the application of 2D low-pass filter is avoided. Instead of applying low-pass filter again on the image domain, which makes the fingerprint ridge block more blurred, a new concept, called 'Ternarization' is used.

To estimate the fingerprint image quality a trained back propagation neural network is used, which contains 11 input nodes, and 1 output node as shown in Fig.1. When the 11 inputs are given for each orientation, the trained neural network returns a value. It responds with large value to correct ridge orientation of ridge block (of high quality or manually recoverable) and responds with a small value to those blocks which contain no ridges or contain manually unrecoverable ridges or falsely estimated orientations. The training method is demonstrated in [12]. 


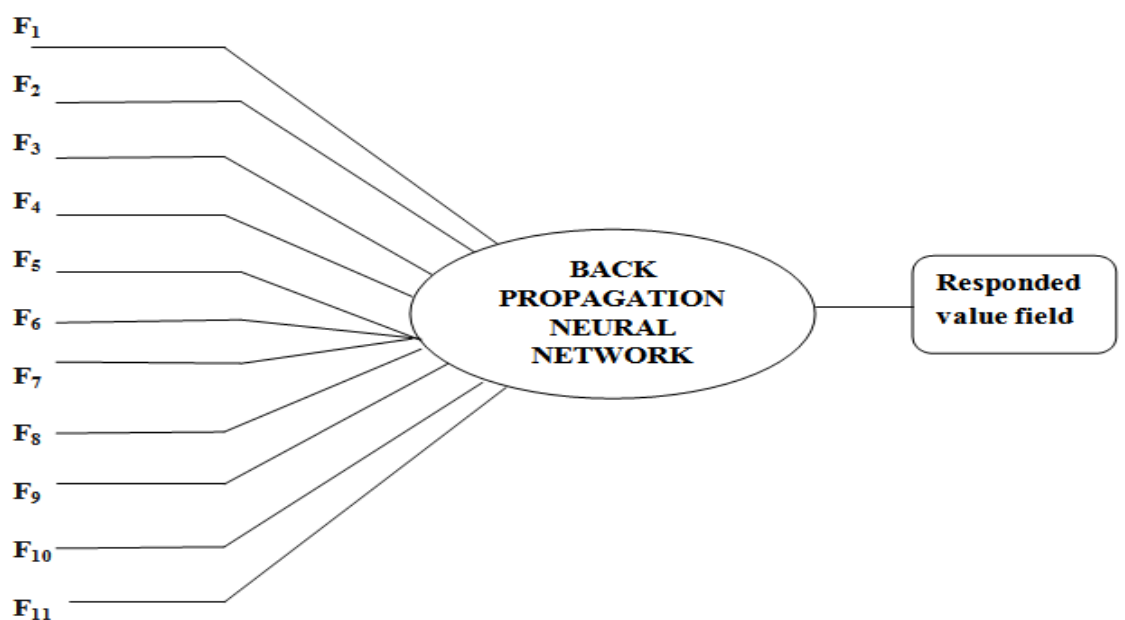

Fig.1 Inputs passed to Neural Network

The trained network would generally respond with large values to the vectors corresponding to the orientation close to the true ridge orientation, and respond with small values to other vectors. These responded values are used to each block to estimate the ridge orientation.

Let I be a gray-level fingerprint image of size $\mathrm{m} \mathrm{x} \mathrm{n}$, where $\mathrm{I}(\mathrm{x}, \mathrm{y})(0<=\mathrm{x}<\mathrm{m}, 0<=\mathrm{y}<\mathrm{n})$ is the intensity of the pixel at $\mathrm{x}^{\text {th }}$ row and $\mathrm{y}^{\text {th }}$ column. Suppose that the image ' $\mathrm{I}$ ' is divided into non-overlapped blocks of size $\mathrm{w}$ $\mathrm{x} \mathrm{w}$

(in the present work the size of the block is $15 \times 15)$ and let $W(i, j)$ denote the block at the $i^{\text {th }}$ row and the $j^{\text {th }}$ column. The center coordination of $\mathrm{W}(\mathrm{i}, \mathrm{j})$ is $(\mathrm{i} \mathrm{xw}+\mathrm{w} / 2, \mathrm{jxw}+\mathrm{w} / 2) . O_{w(i, j)}^{I} \in[0, \Pi]$ is defined as the orientation of $\mathrm{W}(\mathrm{i}, \mathrm{j})$.

And the ridge orientation is quantified into 12 orientations: the kth orientation is $\mathrm{k} \cdot \pi / 12(0 \leq \mathrm{k}<12)$. For each block $\mathrm{W}(\mathrm{i}, \mathrm{j}), 12$ vectors, denoted as $\left\langle\mathrm{F}_{1}, \mathrm{~F}_{2}, \ldots . . \mathrm{F}_{11}\right\rangle^{\mathrm{k}}(0 \leq \mathrm{k}<12)$, can be computed, where $\left\langle\mathrm{F}_{1}, \mathrm{~F}_{2}\right.$, $\ldots . . \mathrm{F}_{11}>{ }^{\mathrm{k}}$ corresponds to the orientation $\mathrm{k} \cdot \pi / 12$.

The process of estimating the orientation field is as follows:

1. Estimate the responded value fields of the image I by the network on each orientation;

2. Filtering the responded value fields in the orientation domain by low-pass filtering;

3. Apply Ternarization to eradicate false bright blocks

4. Orientation selection.

\subsection{Estimate Responded Value Field}

The estimation of the responded value fields of the image I by the network on each orientation is done through process, which described in the below section.

\subsection{Divide Fingerprint Image into Blocks}

A Finger Print image is divided into number of blocks as shown in Fig.2. Each image block, which is also called ridge block in this paper, highlights a local region that is used to isolate the local ridge and valley pattern within the image.

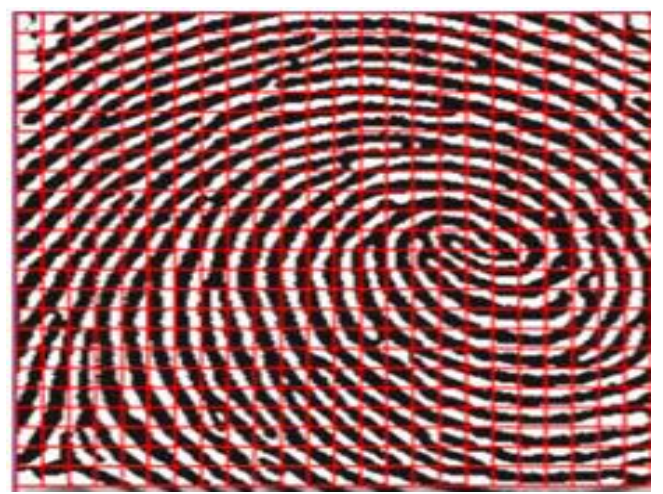

Fig. 2 Fingerprint Image into Blocks 


\subsection{Generate a Feature Vector for Each Block}

Once the fingerprint image is divided into blocks then for each block 11 features, which are denoted as $\left\langle\mathrm{F}_{1}, \mathrm{~F}_{2}, \ldots \ldots \mathrm{F}_{11}\right\rangle$ are computed to give as input to the neural network. Taking these inputs the trained neural network outputs a responding value to indicate whether the estimated local orientation is true or false.

For each image block 11 features, denoted as $\left\langle\mathrm{F}_{1}, \mathrm{~F}_{2}, \ldots \ldots \mathrm{F}_{11}\right\rangle$, are computed to input to the neural network that outputs a responding value to indicate whether the estimated local orientation is correct or not. Let $\left\langle\mathrm{G}_{\mathrm{x}}(\mathrm{u}, \mathrm{v}), \mathrm{G}_{\mathrm{y}}(\mathrm{u}, \mathrm{v})\right\rangle$ be the gradient at the pixel $(\mathrm{u}, \mathrm{v})$. Let $(\mathrm{i}, \mathrm{j})$ be the center of $\mathrm{W}(\mathrm{k}, \mathrm{l})$. The feature vector $\left\langle\mathrm{F}_{1}\right.$, $\mathrm{F}_{2}, \ldots . . \mathrm{F}_{11}>$ for image block $\mathrm{W}(\mathrm{k}, \mathrm{l})$ is computed as described below:

(i) $\mathrm{F}_{1}$, the normal of the sum of the squared gradient vector in the block $\mathrm{W}(\mathrm{k}, \mathrm{l})$, is computed as

$$
F_{1}=\left|\sum_{u=i-\frac{W}{2}}^{i+\frac{W}{2}} \sum_{v=j-\frac{W}{2}}^{j+\frac{W}{2}}\left\langle G_{x}(u, v), G_{y}(u, v)\right\rangle^{2}\right|
$$

(ii) $\mathrm{F}_{2}$, the sum of the normal of the squared gradient vector in the block $\mathrm{W}(\mathrm{k}, \mathrm{l})$, is computed as

$$
F_{2}=\sum_{u=i-\frac{W}{2}}^{i+\frac{W}{2}} \sum_{v=j-\frac{W}{2}}^{j+\frac{W}{2}}\left|\left\langle G_{x}(u, v), G_{y}(u, v)\right\rangle^{2}\right|
$$

(iii) $\mathrm{F}_{3}$, the mean gray of the block $\mathrm{W}(\mathrm{k}, \mathrm{l})$, is computed as

$$
F_{3}=\sum_{u=i-\frac{W}{2}}^{i+\frac{W}{2}} \sum_{v=j-\frac{W}{2}}^{j+\frac{W}{2}} I(u, v) /(w \cdot w)
$$

(iv) $\mathrm{F}_{4}$, the gray variance of the block $\mathrm{W}(\mathrm{k}, \mathrm{l})$ is computed as

$$
F_{4}=\sum_{u=i-\frac{W}{2}}^{i+\frac{W}{2}} \sum_{v=j-\frac{W}{2}}^{j+\frac{W}{2}}\left(I(u, v)-F_{2}\right)^{2} /(w \cdot w-1)
$$

(v) $\mathrm{F}_{5}$ is the variance of the projected signal along the direction orthogonal to the estimated local ridge orientation as shown in Fig.3 (A). The projected window is of size $\mathrm{LxH}$ and with the side L parallel to the estimated local orientation. And the center of the window and center of the block W (k, l) are overlapped. $\mathrm{F}_{5}$ is computed using following equation

$$
F_{5}=\sum_{l=-L / 2}^{L / 2}\left(P_{v}[l]-\sum_{k=-L / 2}^{L / 2} P_{v}[k] / L\right) /(L-1)
$$

Where $P_{w}[k]$ is calculated as follows

$P_{w}[k]=\frac{\sum_{k=-H / 2}^{H / 2} I\left(i-h \cdot \sin \left(O_{w i(i, j)}^{I}\right)+k \cdot \cos \left(O_{w(i, j)}^{I}\right), j+h \cdot \cos \left(O_{w(i, j)}^{I}\right)+k \cdot \sin \left(O_{w(i, j)}^{I}\right)\right)}{H}$

(vi) $\mathrm{F}_{6}$ is the variance of the projected signal along the direction parallel to the estimated local ridge orientation as shown in Fig. 3 (B). The projection window is of size $\mathrm{L} \times \mathrm{H}$ (side $\mathrm{H}$ is parallel to the estimated local orientation) and its center is overlapped with the center of block $\mathrm{W}(\mathrm{k}, \mathrm{l}) . \mathrm{F}_{6}$ is computed using equation (6)

$$
F_{6}=\sum_{l=-L / 2}^{L / 2}\left(P_{v}[l]-\sum_{k=-L / 2}^{L / 2} P_{v}[k] / L\right) /(L-1)
$$

Where $P_{w}[k]$ is calculated as follows 


$$
P_{v}[k]=\frac{\sum_{h=-H / 2}^{H / 2}\left(i-h \cdot \sin \left(O_{w(i, j)}^{I}\right)+k \cdot \cos \left(O_{w(i, j)}^{I}\right), j+h \cdot \cos \left(O_{w(i, j)}^{I}\right)+k \cdot \sin \left(O_{w(i, j)}^{I}\right)\right)}{H}
$$

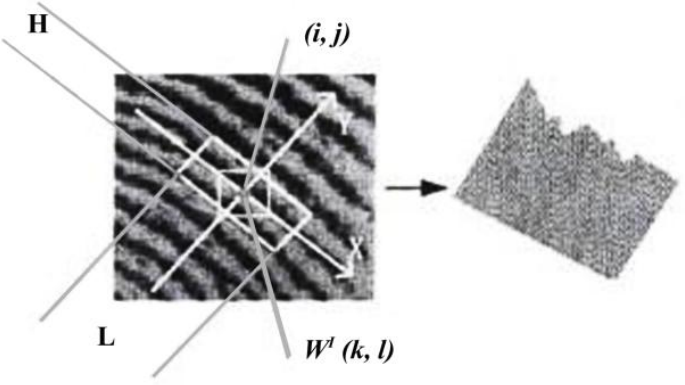

(A)

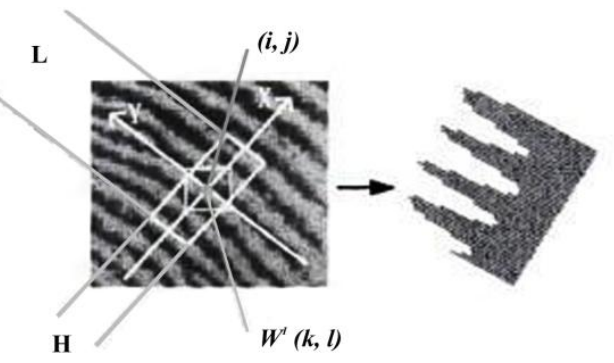

(B)

Fig.3: Projection (A) Along the direction orthogonal to the estimated ridge orientation.

(B) Along the direction parallel to the estimated ridge orientation.

The other features are computed using the smoothed signal, by Gauss filter, of $P h[k](-L / 2 \leq k \leq L / 2)$. Fig.4 gives out an example of computing other features from $\mathrm{F}_{7}$ to $\mathrm{F}_{11}$ based on the smoothened histogram [12].
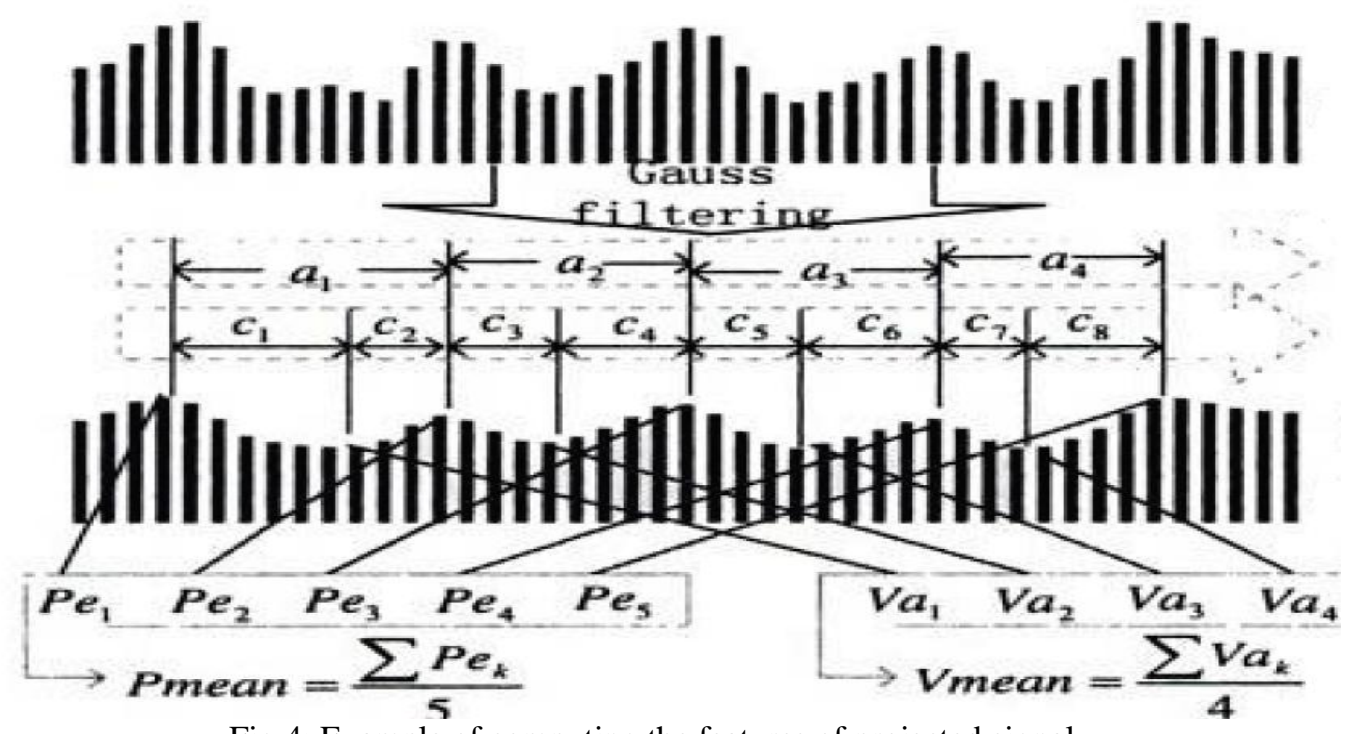

Fig.4: Example of computing the features of projected signal

Based on the above example histogram the following equations are used to find inter ridge distance, variance and of peaks and valleys and also the amplitude of the signal.

(vii) $\mathrm{F}_{7}$, is the average inter-ridge distance

$$
F_{7}=\frac{\sum a_{k}}{4}
$$

(viii) $\mathrm{F}_{8}$, is the variance of the peak heights of the signal

$$
\begin{gathered}
F_{8}=\frac{\sum\left(P e_{k}-P_{\text {mean }}\right)^{2}}{4} \\
\text { Where } P_{\text {mean }}=\frac{\sum P e_{k}}{5}
\end{gathered}
$$

(ix) $\mathrm{F}_{9}$, is the variance of the valley heights of the signal

$$
F_{9}=\frac{\sum\left(V a_{k}-V_{\text {mean }}\right)^{2}}{3}
$$




$$
\text { Where } V_{\text {mean }}=\frac{\sum V a_{k}}{4}
$$

(x) $\mathrm{F}_{10}$, is the average amplitude of the signal

$$
F_{10}=P_{\text {mean }}-V_{\text {mean }}
$$

(xi) $F_{11}$, is the variance of inter-peak-valley distance

$$
\begin{array}{r}
F_{11}=\frac{\sum\left(c_{k}-c_{\text {mean }}\right)^{2}}{7} \\
\text { Where } C_{\text {mean }}=\frac{\sum C_{k}}{8}
\end{array}
$$

As mentioned earlier the trained back propagation neural network is given this derived feature vector as 11 input nodes, and 1 output is produced. The trained back propagation neural network responds to correct ridge orientation of ridge block (of high quality or manually recoverable) with a large value, and responds with a small value to those blocks which contain no ridges or contain manually unrecoverable ridges or are of falsely estimated orientations.

The responded value by the trained network to a specific block is influenced by the ridge orientation, because the items from $F_{5}$ to $F_{11}$ of the input vector $\left\langle F_{1}, F_{2}, \ldots . . F_{11}\right\rangle$ have a close relationship with the estimated ridge orientation. Suppose that the image is divided into un overlapped blocks as shown in Fig.2 and let W (i,j) denote the block at the ith row and the jth column. The ridge orientation is quantified into 12 orientations: the $\mathrm{kth}$ orientation is $\mathrm{k} \cdot \pi / 12(0 \leq \mathrm{k}<12)$. For each block $\mathrm{W}(\mathrm{i}, \mathrm{j}), 12$ vectors, denoted as $\left\langle\mathrm{F}_{1}, \mathrm{~F}_{2}, \ldots \ldots \mathrm{F}_{11}\right\rangle^{\mathrm{k}}(0 \leq \mathrm{k}$ $<12)$, can be computed, $\left\langle\mathrm{F}_{1}, \mathrm{~F}_{2}, \ldots . . \mathrm{F}_{11}>^{\mathrm{k}}\right.$ corresponding to the orientation $\mathrm{k} \cdot \pi / 12$. For each block, feed the 12 vectors to the network and obtain 12 responded values, respectively. The trained network would generally respond with large values to the vectors corresponding to the orientation close to the true ridge orientation, and respond with small values to other vectors.

\subsection{Computation of Responded Value Field}

$\mathrm{R}[\mathrm{k}],(\mathrm{k}=0,1,2, \ldots, 11)$ denotes the responded value field of the image 'I' by the neural network on the kth orientation $(\mathrm{k} \pi / 12)$, and $\mathrm{R}[\mathrm{k}](\mathrm{i}, \mathrm{j})=\mathrm{N}(\mathrm{W}(\mathrm{i}, \mathrm{j}), \mathrm{k})$ represents the responded value by the network to the block $\mathrm{W}(\mathrm{i}, \mathrm{j})$ (ith row and jth column) on the kth orientation. Fig. 4 gives out the gray representation of responded value field of a sample fingerprint image, on the 12 quantified orientations $(0, \pi / 12, \pi / 6, \pi / 4, \pi / 3,5 \pi / 12$, $\pi / 2,7 \pi / 12,8 \pi / 12,9 \pi / 12,10 \pi / 12$ and $11 \pi / 12)$.

For a responded value field on a certain orientation is shown in Fig.5, the white block indicates that it is fairly possible that the corresponding ridge block is of the correct orientation.

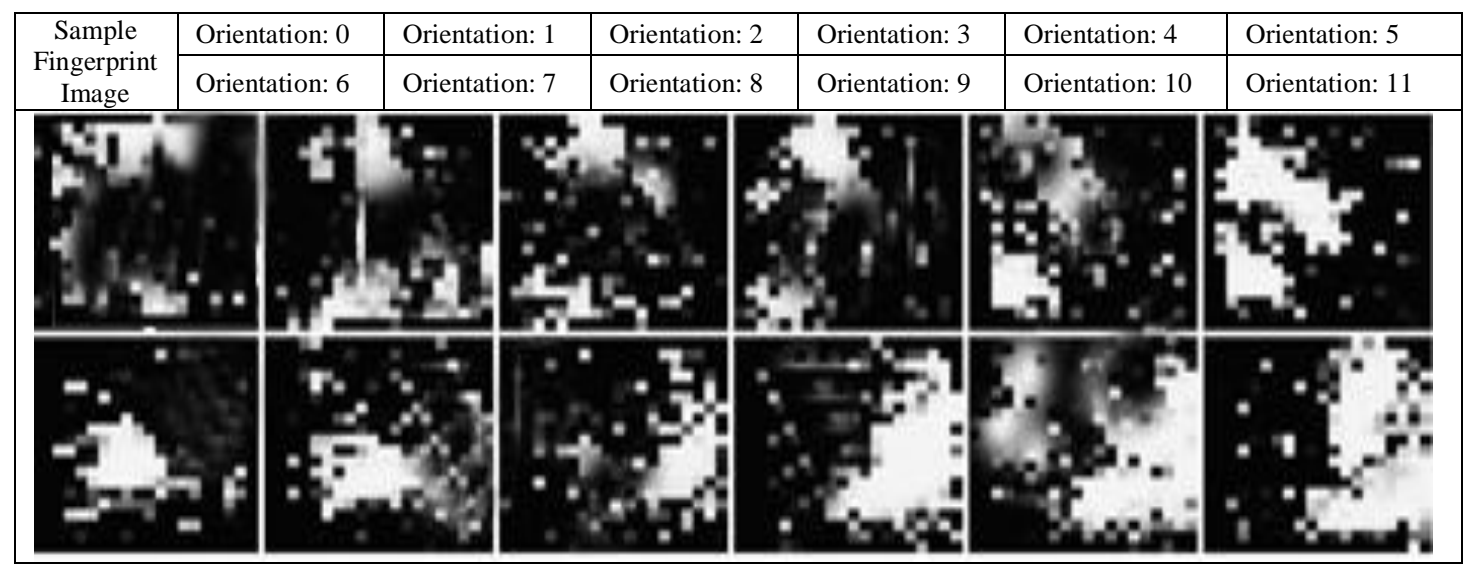

Fig.5: The responded value field by the network on 12 orientations of the image.

\subsection{Filtering on Orientation Domain}

It is observed in the experimental session that some low quality ridge blocks are of large responded value on some false orientations. And this is reflected by the isolated bright blocks in Fig.5. These false orientations of large responded value are usually isolated and thus the isolated large value can be repressed by low-pass filtering on orientation domain. The responded values, $\mathrm{R}[\mathrm{k}](\mathrm{i}, \mathrm{j})(\mathrm{k}=0,1,2, \ldots, 11)$, of the block $\mathrm{W}(\mathrm{i}, \mathrm{j})$ on different orientations is filtered as shown in Equation (1), where $\omega(\mathrm{u})$ is the low-pass filter, and get $\mathrm{R}^{\prime}[\mathrm{k}](\mathrm{i}, \mathrm{j})$. 
Fig. 6 shows the low-pass filtered results of original responded value fields in Fig. 5 after applying the low pass filter.

$$
R^{\prime}[k](i, j)=\sum_{u=-1}^{1} w(u) \cdot R[k+u](i, j)
$$
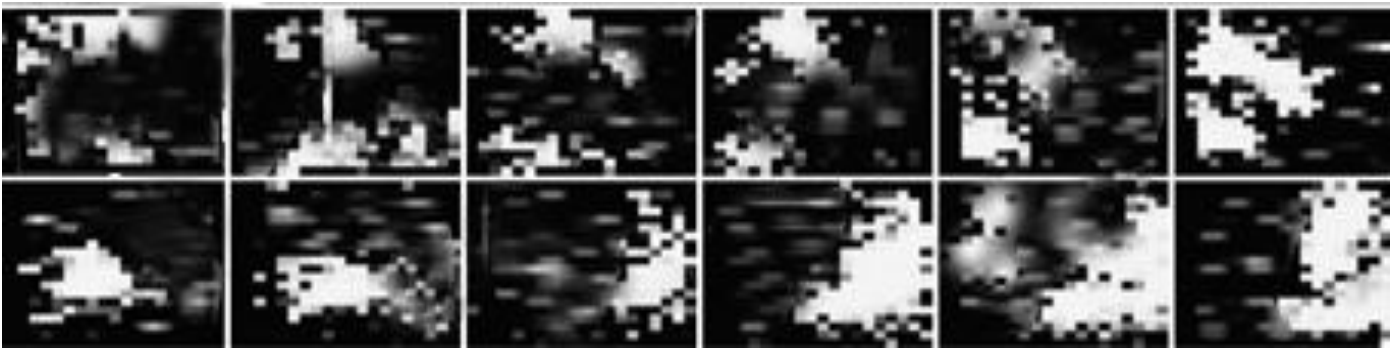

Fig.6: Low-pass filtered results, of fields in Fig. 5, on the orientation domain

\subsection{Filtering in Image Block Domain Vs Ternarization}

As shown by Fig.6, the low-pass filtered responded field is still not smooth enough that some bright blocks are surrounded by black blocks and some black blocks are surrounded by bright blocks. However fingerprint ridge orientation usually changes smoothly between neighbor blocks. Hence, in [12] the low-pass filtered fields were again filtered using 2-dimensional low-pass filter on the image block domain so that the filtered responded values changes smoothly between neighbor blocks, which is accordant with the ridge orientation changing. Equation (13) gives the process of low-pass filtering on the image block domain, where $\mathrm{R}^{\prime \prime}[\mathrm{k}](\mathrm{i}, \mathrm{j})$ denotes the low-passed value of the block $\mathrm{W}(\mathrm{u}, \mathrm{v})$ on the kth orientation Fig. 7 shows the low-pass filtered results of Fig. 6

$$
R^{n}[k](i, j)=\sum_{u=-1}^{1} \sum_{v=-1}^{1} w(u, v) \cdot R^{z}[k](i+u, j+v)
$$
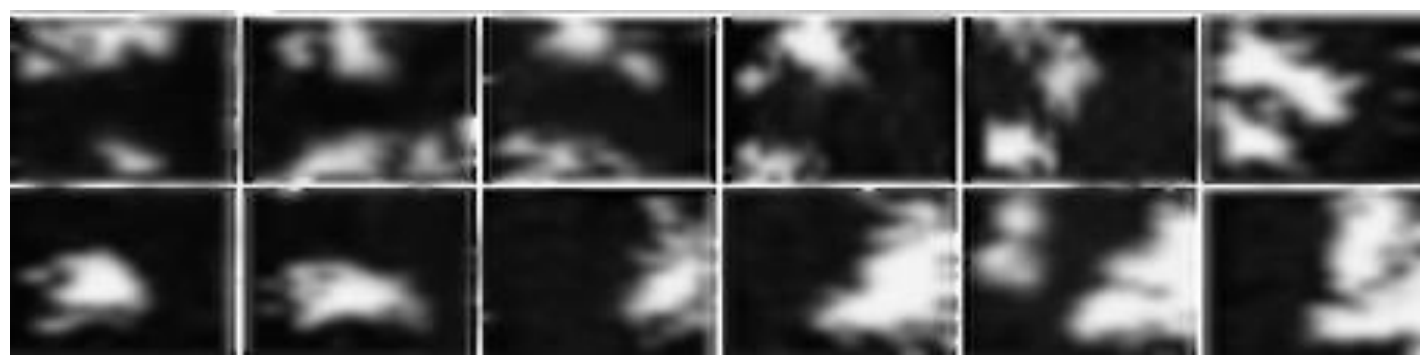

Fig.7: Low-pass filtered results, of images in Fig.6, on the image block domain.

It can be observed from the above Figure that after applying the two dimensional low-pass filter the orientation image has become too much blurred. Even though the isolated bright parts are eliminated, the bright ridge blocks (which indicate that it is fairly possible that the corresponding ridge blocks are of the correct orientation) have become more blurred and this causes problem during the minutiae extraction. So this approach has been modified in the present work; instead of applying again a 2D low-pass filter on the image domain, a new process, which is called Ternarization, is introduced, which is described in the following section.

\subsection{Ternarization}

Instead of dividing into two blocks as in segmentation, here three blocks are considered, they are

- White block: which consists the ridge blocks with true ridge orientation with high responded value by the neural network (white part in the Figure.)

- Black block: which consists the false orientation with low responded value (black part in the Figure.)

- Gray block: which consists the blocks with uncertainty (probably the false orientation with high responded value.

The fingerprint image is divided based on the variance in the gray level intensity. Two threshold values $T_{1}, T_{2}$ are taken here. Wherein $T_{1}$ is the low end threshold value and $T_{2}$ is the high end threshold value. If the variance is less than $T_{1}$, it is assigned to the black block and these are of no interest and can be eliminated. If 
the variance is greater than the threshold $T_{2}$ it is then assigned it to the white block. These are the bright parts, whose corresponding ridge block is of the correct orientation. Now if the variance is neither less than $T_{1}$ nor greater than $T_{2}$ then it is assigned to the gray block. This gray block indicates uncertainty, which needs to be handled properly.

Some low quality ridge blocks are of large responded value on some false orientations. And this is reflected by the isolated bright blocks, which are shown in Fig.5. These false orientations of large responded value are usually isolated [12]. These isolated large values were repressed by low-pass filtering on orientation domain.

It can be observed from the figure that the isolated bright parts are not as bright as the white block regions. This is the result of applying the Gaussian filter on the orientation domain. So these are the ones that are placed in the gray list.

This gray list must become empty by throwing its elements either into white block or black block through using a distance measure. A distance threshold ' $\mathrm{D}$ ' is used to calculate the distance between the bright block elements and gray block elements: if the distance is less than the threshold ' $\mathrm{D}$ ' then the element is assigned to white block, which is the bright block that reflects the true ridge orientation. If the distance is greater than ' $\mathrm{D}$ ' then it indicates that it is an isolated bright block and thus reflects a false orientation which was assigned a large value. Hence these isolated bright blocks, whose distance is greater than the threshold ' $D$ ' should be assigned to black block and have to be eliminated

Fig. 8 Shows the results of original responded value fields after applying the Ternarization process on the low pass filtered results, which are shown in Fig.6
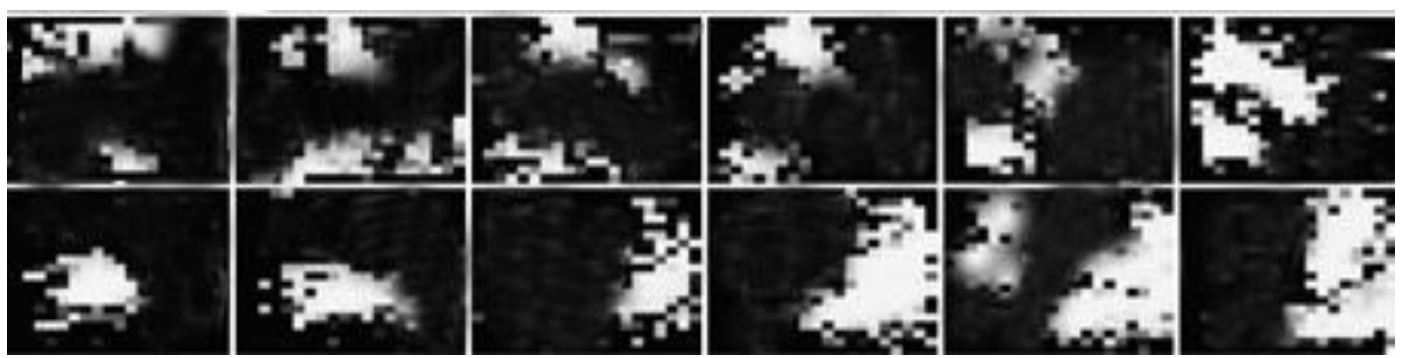

Fig.8 Result after Ternarization on the filtered results, of images in Fig.6

\subsection{Orientation Selection}

Each image block has 12 responded values which are filtered on orientation domain and Ternarization, and each value is corresponding to one of the12 quantified orientations. The orientation selection for each image block is made based on the orientation corresponding to the largest out of the 12 resulted values. This is shown in Equations (14) and (15). Where $\operatorname{TR}^{\prime}[\mathrm{k}]\left(i_{,}, j\right)$ denotes value of the block $\mathrm{W}(\mathrm{u}, \mathrm{v})$ after the Ternarization is applied on $\mathrm{R}^{p}[\mathrm{k}](i, j)$.

$$
\begin{gathered}
\operatorname{TR}^{\prime}[\mathrm{k}](i, j)=\max \left(\operatorname{TR}^{\prime}[l](\mathrm{i}, \mathrm{j}), \quad \text { where } 0 \leq l \leq 11\right) \\
\mathrm{o}(\mathrm{W}(\mathrm{i}, \mathrm{j}))=\mathrm{k} \cdot \frac{\pi}{12}
\end{gathered}
$$

\section{Experimental Results}

It is hard to evaluate the quality of a method for estimating orientation field. The quality of orientation estimation can be indirectly measured by computing the accuracy of feature detection and matching accuracy.

However, aside from orientation estimation, these quality measures and matching accuracy are also dependent on other enhancement processes. So evaluating the accuracy of ridge orientation estimation alone in the enhancement process is a tough task.

Of course computing the deviation between the orientation field by the proposed method and the one manually labeled can also be used for evaluating the orientation field estimation method. Yet manually labeling the orientation fields of a good many fingerprint images is an exhausting work, and it is of no necessity, since the results of orientation estimation by two different methods can be obviously compared by visually inspection.

To evaluate the effectiveness of the proposed technique of ridge orientation estimation, a combination of both synthetic test images and real fingerprint images were used in the experiments. 


\subsection{Synthetic test image results}

The first sets of experiments were conducted on a set of circular synthetic images. By using synthetic images, the pre-determined orientation values can serve as a basis for comparison with the orientation values computed by the orientation estimation algorithm. The accuracy of the algorithm is quantitatively measured by the mean square error between the estimated and actual values. The mean square error represents the difference between the actual orientation values and the estimated orientation values in radians.

The algorithm was first tested on a number of well-defined synthetic images. The results were achieved using different image sizes and wavelengths to construct the synthetic images. Visual inspection of these results shows that the estimated orientation field is smooth and well defined throughout the image. Furthermore, the small mean square error values indicate that there is minimal difference between the actual orientation values and the estimated values from the proposed method. Thus, it can be shown that the algorithm is able to produce accurate orientation estimates for synthetically well-defined images.

Experiments were then conducted with a series of random noisy elements applied to the images. The Gradient based approach [13-17] for estimating the orientation and the present method of estimating the orientation are compared. Fig. 9(c) shows that in Gradient based approach, the presence of small amounts of noise does not significantly affect the accuracy of the algorithm. However, Fig. 9(f) illustrates that when higher intensities of noise are applied to the image, Gradient based algorithm produces a disordered orientation field with a high proportion of misaligned orientation vectors. Fig. 9 illustrates that even with higher intensities of noise the proposed method produces an accurate estimation of ridge orientation

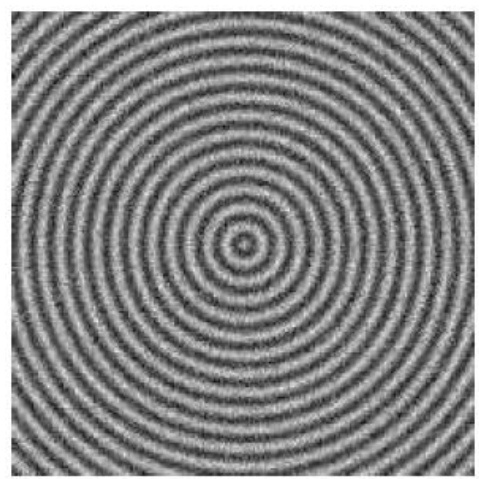

(a) Original image

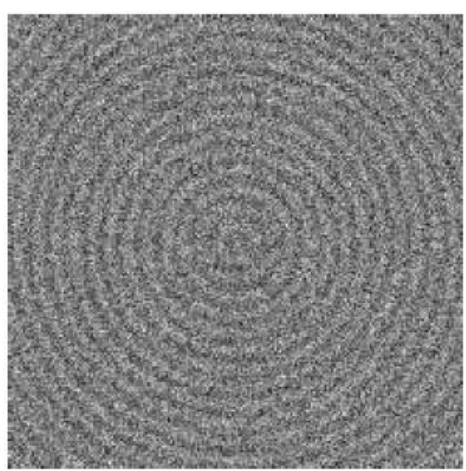

(d) Original image

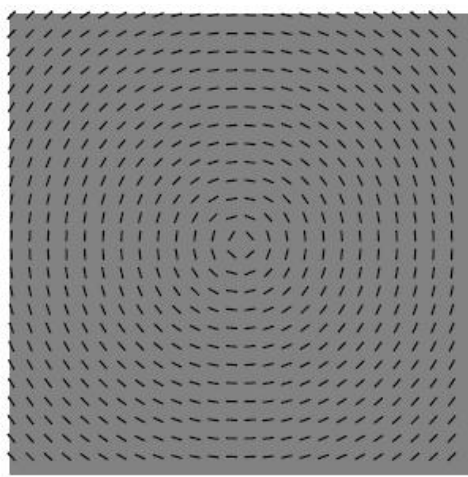

(b) Orientation image

(Gradient based)

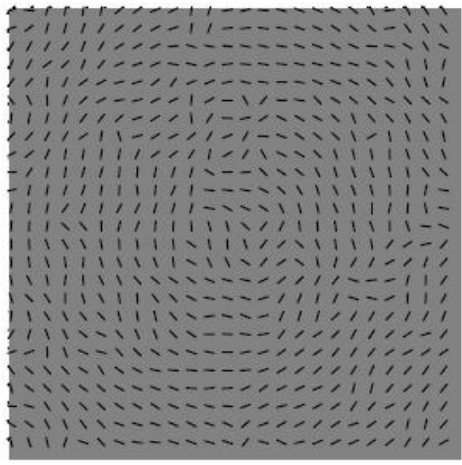

(e) Orientation image (Gradient based)

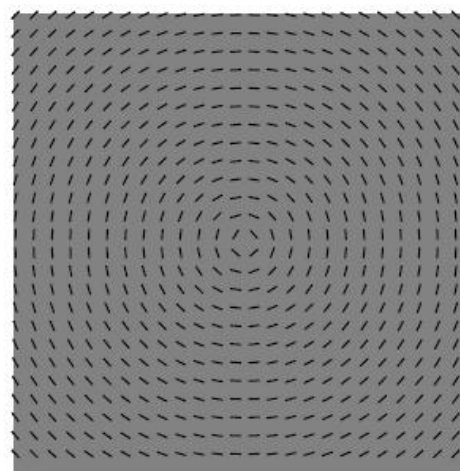

(c) Orientation image

(Proposed Method)

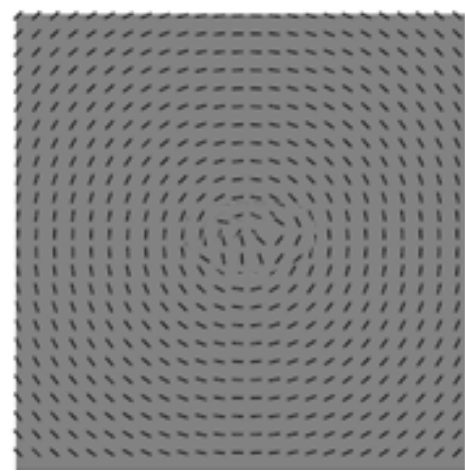

(f) Orientation image

(Proposed Method)

Fig.9: The estimated orientation for 200X200 synthetic images of wavelength 8. Random noise with standard deviation values of 0.5 (top row), and 3.0 (bottom row) are applied to the images, respectively.

It can be observed from the Fig. 9 that, for an image of good quality both the gradint based and proposed method produced correct ridge orientation but the gradient based method failed to produce correct orientation when the given input image is noisy. The proposed method produced correct orientation even for the noisy image, except in the center where the noise is too much. Table 1 provides the Mean Square Error (in terms of radians) of the two methods at various standard deviations. 
Table 1: The mean square error values (radians) for varying intensities of noise. The noise is applied to a 200 X 200 sized synthetic image of wavelength 8 .

\begin{tabular}{|c|c|c|}
\hline $\begin{array}{c}\text { Standard } \\
\text { Deviation }\end{array}$ & $\begin{array}{c}\text { Mean Square Error } \\
\text { (Gradient Based Method) }\end{array}$ & $\begin{array}{c}\text { Mean Square Error } \\
\text { (Proposed Method) }\end{array}$ \\
\hline 0.0 & 0.0003 & 0.00001 \\
0.5 & 0.0009 & 0.0001 \\
1.0 & 0.0032 & 0.0008 \\
1.5 & 0.0102 & 0.0021 \\
2.0 & 0.0246 & 0.0098 \\
2.5 & 0.0691 & 0.0212 \\
3.0 & 0.1722 & 0.0791 \\
3.5 & 0.2330 & 0.1023 \\
4.0 & 0.3041 & 0.1754 \\
4.5 & 0.4124 & 0.2032 \\
5.0 & 0.4262 & 0.2352 \\
\hline
\end{tabular}

Table 1 shows the mean square error values with respect to increasing values of noise intensity when applied to a 200 X200 sized synthetic image of wavelength eight. The large mean square errors indicate that the accuracy of the algorithm decreases significantly in the presence of high intensities of noise. Therefore, it can be shown that the gradient based algorithm can produce accurate orientation estimates in the presence of minimal amounts of noise, but its performance deteriorates under high levels of noise. But the proposed method could produce accurate orientation estimation even in the presence of noise.

\subsection{Real Fingerprint Image Results}

The second set of experiments was conducted on a series of real fingerprint images. A diverse mixture of fingerprint images was selected to assess the algorithm's performance under various fingerprinting conditions. In order to quantitatively compare the two methods, A and B, the number of blocks of incorrect orientation are counted. 5 images are used in the experiments. And for each image, the number of blocks of incorrect orientation is shown in Table 2 which shows that method B performs much better than method A.

The five images, that are used in the experiment for orientation estimation and results of those 5 images by the two methods, A (left column) and B (right column) are presented at the end.

Two methods are compared for estimating fingerprint orientation field: method A-Gradient based method

[13-17] followed by Gaussian low pass filtering [18]; method B - the proposed method

Table 2: Block counts of incorrect orientation

\begin{tabular}{|c|c|c|}
\hline \multirow{2}{*}{ Image Name } & \multicolumn{2}{|c|}{$\begin{array}{c}\text { The number of ridge blocks of incorrect } \\
\text { arientation }\end{array}$} \\
\cline { 2 - 3 } & $\begin{array}{c}\text { Method A } \\
\text { (Gradient based) }\end{array}$ & $\begin{array}{c}\text { Method B } \\
\text { (Proposed Method) }\end{array}$ \\
\hline Image 1 & 25 & 0 \\
\hline Image 2 & 18 & 3 \\
\hline Image 3 & 10 & 2 \\
\hline Image 4 & 60 & 15 \\
\hline Image 5 & 62 & 0 \\
\hline
\end{tabular}

Table 2 shows the results of both gradient based and proposed method in terms of "number of ridge blocks of incorrect orientation' when a fingerprint image is given. It can be observed that the proposed method produced very less number of incorrect orientations comparing with the gradient based approach of estimating ridge orientation. So the experimental results show that both with synthesis images and real images results, the proposed method outperforms the existing traditional gradient based method for estimating ridge orientation.

These results in Table 2 are obtained on standard five sample images which were used for the similar works in the literature. The orientation estimation results of the fifth sample image by the two methods, Gradient based method followed by Gaussian low pass filtering 'A' (left column) and the proposed method 'B' (right column) are presented figure 10 . 

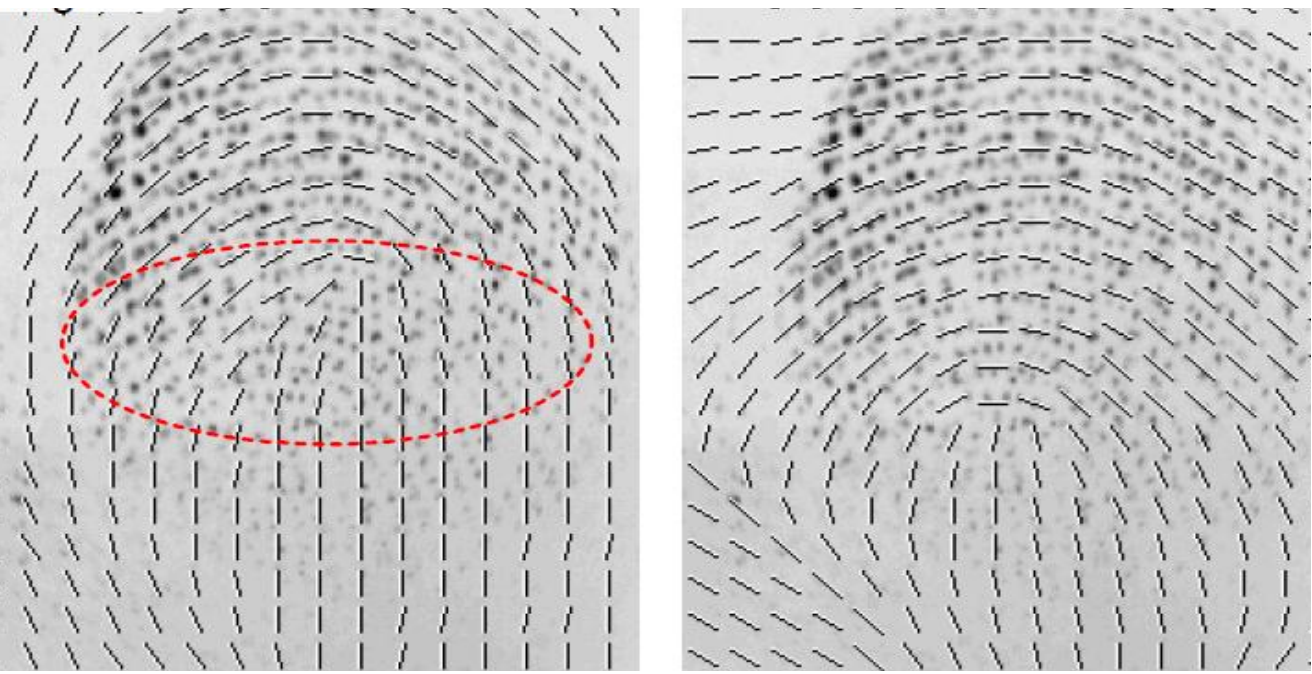

Fig. 10: Comparison of orientation estimation between method A (left column) and method B (right column). The dotted circles indicate that the orientations in circled region are incorrect.

More experiments are also conducted on very poor quality fake fingerprints of the ATVS-FFp DB database. Twenty sample results are presented in the graph in figure. In most of the fingerprints that were considered has only portion of the ridge structure. Twenty sample results out of many experiments are presented in Fig.11.

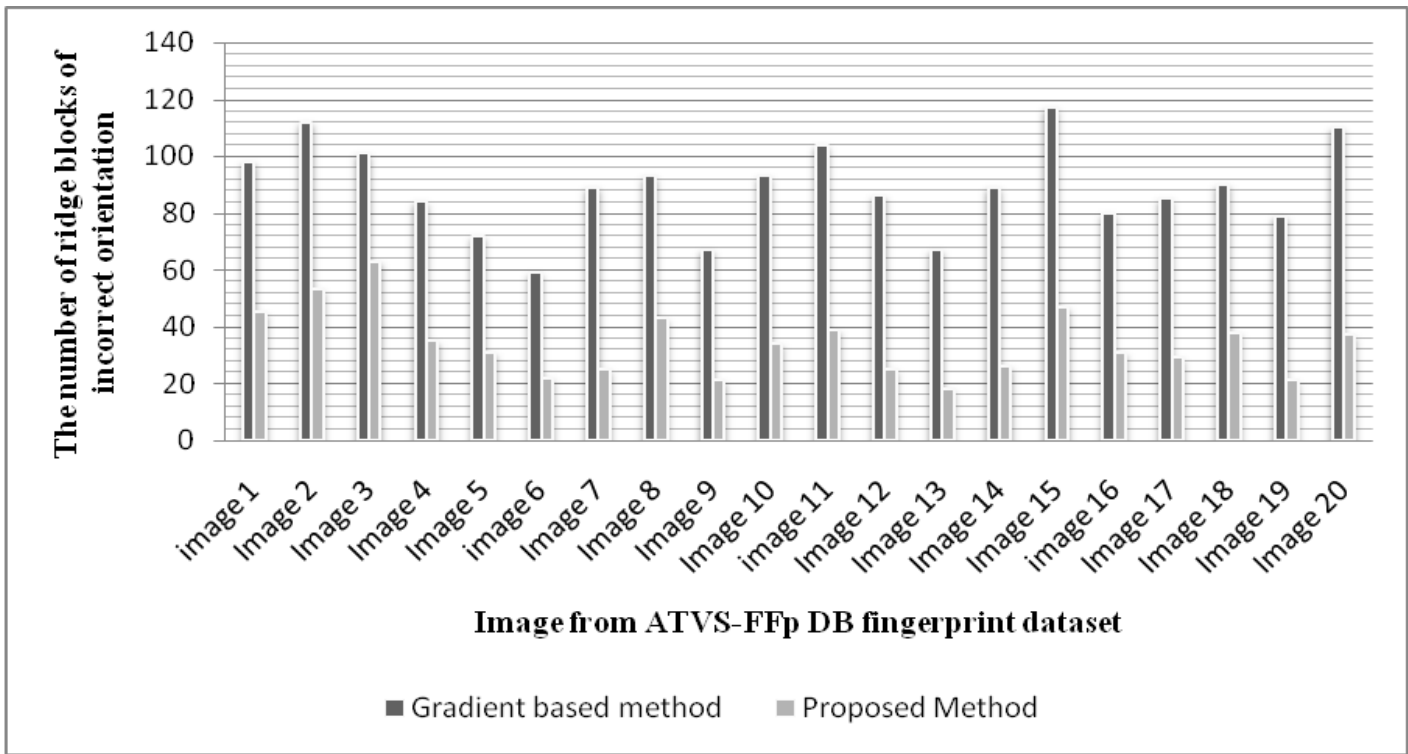

Fig.11: The number of ridge blocks of incorrect orientation - comparative results between Gradient based approach and proposed method.

It is very clear from the graph in Fig.10 that the proposed method for estimating ridge orientation works far better than the traditional gradient based approach. With the proposed methodology the number of ridge blocks of incorrect orientation is comparatively very less even with the noisy poor quality fingerprint images.

\section{Conclusions}

Ridge orientation estimation is a necessary and fundamental step in fingerprint image enhancement and recognition. This paper describes a method for fingerprint ridge orientation estimation using neural network approach and Ternarization. The best orientation was selected based on the responded value by the trained back propagation neural network and these responded fields are refined through the process of Ternarization.

Experiments show that the proposed method leads to a greater improvement in ridge orientation estimation over the other Gradient based approaches. 


\section{References}

[1] Zhu E., Yin J.P., Zhang G.M., Fingerprint Matching Based on Global Alignment of Multiple Reference Minutiae, Pattern Recognition, 2005, 38(10): 1685-1694.

[2] Zhu E., Yin J.P., Zhang G.M., Hu C.F., Fingerprint Minutia Relationship Representation Proceedings of the 5th WSEAS Int. Conf. on Signal Processing, Robotics and Automation, Madrid, Spain, February 15-17, 2006 (pp158-164) and Matching Based on Curve Coordinate System, International Journal of Image and Graphics, 2005, 5(4): 729-744.

[3] Kawagoe M., Tojo A., Fingerprint Pattern Classification, Pattern Recognition, 1984, 17(3): 295-303.

[4] Mehtre B.M., Murthy N.N., Kapoor S., Segmentation of Fingerprint Images Using the Directional Image, Pattern Recognition, 1987, 20(4): 429-435.

[5] Hung D.C.D., Enhancement and Feature Purification of Fingerprint Images, Pattern Recognition, 1993, 26(11): 1661-1672.

[6] En Zhu, Jian-Ping Yin, Guo-Min Zhang, Chun-Feng Hu., "Fingerprint Ridge Orientation Estimation Based on Neural Network", in ISPRA'06 Proc. 5th WSEAS Int. Conference on Signal Processing, Robotics and Automation,2006,pp.158-164.

[7] Sherlock B., Monro D., “A Model for Interpreting Fingerprint Topology”, Pattern Recognition, vol.26, no.7, pp.1047-1055,1993.

[8] Vizcaya P., Gerhardt L., “A Nonlinear Orientation Model for Global Description of Fingerprints”,Pattern Recognition, 1996 , vol. 29, no. 7, pp.1221-1231.

[9] Araque J., Baena M., Chalela B., Navarro D., Vizcaya P., "Synthesis of Fingerprint Images",in Proc. Int. Conf. on Pattern Recognition, 2002,vol. 2,pp. 422-425.

[10] Zhu E., Yin J. P., Hu C. F., Zhang G. M., "Quality estimation of fingerprint image based on neural network", in Proc. Of International Conference on Natural Computing, LNCS 3611, (2005) pp.65-70.

[11] Kovesi, P. MATLAB functions for computer vision and image analysis. School of Computer Science and Software Engineering, The University of Western Australia, [Online]. Available: http://www.cs.uwa.edu.au/»pk/Research/MatlabFns/index.html Accessed: 20 August 2003.

[12] En Zhu, Jianping Yin, Chunfeng Hu, Guomin Zhang, "Quality Estimation of Fingerprint Image Based on Neural Network", Advances in Natural Computation Lecture Notes in Computer Science Volume 3611, 2005, pp 65-70.

[13] Lam L, Lee S W, Suen C Y. Thinning methodologies - a comprehensive survey [J]. IEEE Trans. Pattern Anal. Mach. Intell., 1992, 14 (9): 869-885.

[14] B. M. Mehtre, "Fingerprint image analysis for automatic identification," Machine Vision and Applications 6, 124 -139 (1993).

[15] B. G. Sherlock, D. Monro, and K. Millard, "Fingerprint enhancement by directional fourier filtering," IEE Proceedings of Visual and Image Signal Processing 141(2), (April 1994).

[16] A. Sherstinsky and R.W. Picard. Restoration and enhancement of fingerprint images using m-lattice: A novel non-linear dynamical system. In Proc. $12^{\text {th }}$ ICPR-B, pages 195-200, 1994.

[17] Lee H C, Gaensslen R E. Advances in Fingerprint Technology [M]. Boca Raton: CRC Press, 1994.

[18] L. Hong, Y. Wan, and A. Jain, "Fingerprint image enhancement: algorithms and performance evaluation," IEEE Transactions on Pattern Analysis and Machine Intelligence 20(8), 777 -789 (August 1998).

[19] M. James Stephen and P. V. G. D. Prasad Reddy, Enhancing Fingerprint Image through Ridge Orientation, with Neural Network Approach and Ternarization for Effective Minutiae Extraction, International Journal of Machine Learning and Computing, Vol. 2, No. 4, August 2012

[20] M. James Stephen, Prasad Reddy PVGD, 'Fingerprint Image Enhancement through Particle Swarm Optimization'. International Journal of Computer Applications 66(21):34-40, March 2013. Published by Foundation of Computer Science, New York, USA

[21] M. James Stephen, Prasad Reddy PVGD, et al./ Removal of False Minutiae with Fuzzy Rules from the Extracted Minutiae of Fingerprint Image, Advances in Intelligent and Soft Computing,- Springer, Jan 2012, vol 132/2012, pp. 853-860, 2012.

[22] M. James Stephen, Prasad Reddy PVGD, "Implementation of Easy Fingerprint Image Authentication with Traditional Euclidean and Singular Value Decomposition Algorithms” Int. J. Advance. Soft Comput. Appl., Vol. 3, No. 2, July 2011, ISSN 2074-8523; Copyright @ ICSRS Publication, 2011 